\title{
Remarks on the sequential effect algebras*
}

\author{
Shen Jun ${ }^{1,2}$, Wu Junde ${ }^{1 \dagger}$ \\ ${ }^{1}$ Department of Mathematics, Zhejiang University, Hangzhou 310027, P. R. China \\ ${ }^{2}$ Department of Mathematics, Anhui Normal University, Wuhu 241003, P. R. China
}

\begin{abstract}
In this paper, first, we answer affirmatively an open problem which was presented in 2005 by professor Gudder on the sub-sequential effect algebras. That is, we prove that if $(E, 0,1, \oplus, \circ)$ is a sequential effect algebra and $A$ is a commutative subset of $E$, then the sub-sequential effect algebra $\bar{A}$ generated by $A$ is also commutative. Next, we also study the following uniqueness problem: If $n a=n b=c$ for some positive integer $n \geq 2$, then under what conditions $a=b$ hold? We prove that if $c$ is a sharp element of $E$ and $a \mid b$, then $a=b$. We give also two examples to show that neither of the above two conditions can be discarded.
\end{abstract}

Key Words. Sub-sequential effect algebras, commutative, uniqueness.

\section{Introduction}

Effect algebra is an important logic model for studying quantum effects or observations which may be fuzzy or unsharp (see [1]), to be precise, an effect algebra is a system $(E, 0,1, \oplus)$, where 0 and 1 are distinct elements of $E$ and $\oplus$ is a partial binary operation on $E$ satisfying:

(EA1) If $a \oplus b$ is defined, then $b \oplus a$ is defined and $b \oplus a=a \oplus b$.

*This project is supported by Natural Science Found of China (10771191 and 10471124).

${ }^{\dagger}$ Corresponding author: wjd@zju.edu.cn 
(EA2) If $a \oplus(b \oplus c)$ is defined, then $(a \oplus b) \oplus c$ is defined and

$$
(a \oplus b) \oplus c=a \oplus(b \oplus c)
$$

(EA3) For each $a \in E$, there exists a unique element $b \in E$ such that $a \oplus b=1$.

(EA4) If $a \oplus 1$ is defined, then $a=0$.

In an effect algebra $(E, 0,1, \oplus)$, if $a \oplus b$ is defined, we write $a \perp b$. For each $a \in E$, it follows from (EA3) that there exists a unique element $b \in E$ such that $a \oplus b=1$, we denote $b$ by $a^{\prime}$. Let $a, b \in E$, if there exists an element $c \in E$ such that $a \perp c$ and $a \oplus c=b$, then we say that $a \leq b$ and write $c=b \ominus a$. It follows from [1] that $\leq$ is a partial order of $(E, 0,1, \oplus)$ and satisfies that for each $a \in E, 0 \leq a \leq 1, a \perp b$ if and only if $a \leq b^{\prime}$.

Let $(E, 0,1, \oplus)$ be an effect algebra and $a \in E$. If $a \wedge a^{\prime}=0$, then $a$ is said to be a sharp element of $E$. The set $E_{s}=\left\{x \in E \mid x \wedge x^{\prime}=0\right\}$ is called the set of all sharp elements of $E$ (see [2-3]).

As we knew, two measurements $a$ and $b$ cannot be performed simultaneously in general, so they are frequently executed sequentially ([4]). We denote by $a \circ b$ a sequential measurement in which $a$ is performed first and $b$ second and call $a \circ b$ a sequential product of $a$ and $b$. Thus, it is an important and interesting project to study effect algebras which have a sequential product $\circ$ with some nature properties. To be precise:

A sequential effect algebra (SEA) is an effect algebra $(E, 0,1, \oplus)$ and another binary operation $\circ$ defined on $(E, 0,1, \oplus)$ satisfying [5]:

(SEA1) The map $b \mapsto a \circ b$ is additive for each $a \in E$, that is, if $b \perp c$, then $a \circ b \perp a \circ c$ and $a \circ(b \oplus c)=a \circ b \oplus a \circ c$.

(SEA2) $1 \circ a=a$ for each $a \in E$.

(SEA3) If $a \circ b=0$, then $a \circ b=b \circ a$.

(SEA4) If $a \circ b=b \circ a$, then $a \circ b^{\prime}=b^{\prime} \circ a$ and for each $c \in E, a \circ(b \circ c)=(a \circ b) \circ c$.

(SEA5) If $c \circ a=a \circ c$ and $c \circ b=b \circ c$, then $c \circ(a \circ b)=(a \circ b) \circ c$ and $c \circ(a \oplus b)=(a \oplus b) \circ c$ whenever $a \perp b$. 
Let $(E, 0,1, \oplus, \circ)$ be a sequential effect algebra. If $a, b \in E$ and $a \circ b=b \circ a$, then we say $a$ and $b$ is sequentially independent and denoted by $a \mid b$.

Lemma $1([\mathbf{1}, \mathbf{5}])$. If $(E, 0,1, \oplus, \circ)$ is a sequential effect algebra and $a, b, c \in E$, then

(1) $a \perp b, a \perp c$ and $a \oplus b=a \oplus c$ implies that $b=c$.

(2) $a \in E_{s}$ if and only if $a \circ a=a$.

(3) If $c \in E_{s}$, then $a \leq c$ if and only if $a=a \circ c=c \circ a$.

\section{Sub-sequential effect algebra generated by a subset}

Let $(E, 0,1, \oplus, \circ)$ be a sequential effect algebra and $F$ a nonempty subset of $E$. We call $F$ a sub-sequential effect algebra of $(E, 0,1, \oplus, \circ)$ if $0,1 \in F$ and $(F, 0,1, \oplus, \circ)$ itself is a sequential effect algebra. From the definition of sub-sequential effect algebra, it is easy to see that a nonempty subset $F$ of $(E, 0,1, \oplus, \circ)$ is a sub-sequential effect algebra if and only if $F$ is closed under all the three operations $\oplus$, $\circ$ and '. Moreover, if $A$ is a nonempty subset of $E$, it is easy to see that there exists a smallest sub-sequential effect algebra $\bar{A}$ of $E$ which contains $A$ (That is, the intersection of all sub-sequential effect algebras containing $A$ ). We call $\bar{A}$ the sub-sequential effect algebra generated by $A$. In 2005, Professor Gudder presented the following open problem (see [6, Problem 17]):

Problem 1. If $(E, 0,1, \oplus, \circ)$ is a sequential effect algebra and $A$ a commutative subset of $E$ (That is, $a \mid b$ for all $a, b \in A$ ), is $\bar{A}$ commutative ?

In this paper, we answer the problem affirmatively. That is:

Theorem 1. Let $(E, 0,1, \oplus, \circ)$ be a sequential effect algebra and $A$ a commutative subset of $(E, 0,1, \oplus, \circ)$. Then $\bar{A}$ is also commutative.

Proof. Let $\Lambda=\{F \mid F$ be a commutative subset of $E$ containing $A\}$. We order $\bigwedge$ by including. Using Zorn's Lemma, it is easy to see that there exists a maximal element $F_{0}$ in $\bigwedge$. That is, $F_{0}$ is a maximal commutative subset of $E$ containing $A$.

We now prove that $F_{0}$ is a sub-sequential effect algebra of $E$ : 
If $a \in F_{0}$, then for each $c \in F_{0}, c \mid a$, so $c \mid a^{\prime}$ by (SEA4). By maximality, we have $a^{\prime} \in F_{0}$.

If $a, b \in F_{0}$, then for each $c \in F_{0}, c|a, c| b$, so $c \mid(a \circ b)$ by (SEA5). By maximality, we have $(a \circ b) \in F_{0}$.

If $a, b \in F_{0}$ and $a \perp b$, then for each $c \in F_{0}, c|a, c| b$, so $c \mid(a \oplus b)$ by (SEA5). By maximality, we have $(a \oplus b) \in F_{0}$.

So $F_{0}$ is closed under all the three operations $\oplus$, o and '.

Thus, $F_{0}$ is a sub-sequential effect algebra of $(E, 0,1, \oplus, \circ)$ containing $A$. Since $\bar{A}$ is the smallest sub-sequential effect algebra of $(E, 0,1, \oplus, 0)$ containing $A$, we have $\bar{A} \subseteq F_{0}$ and $\bar{A}$ is also commutative.

Moreover, for general subset $A$ of $E$, we can describe the structure of $\bar{A}$, that is

Theorem 2. Let $(E, 0,1, \oplus, \circ)$ be a sequential effect algebra and $A$ a subset of E. If we denote

$$
\begin{aligned}
& A_{1}=A \cup\left(\bigcup_{a \in A} a^{\prime}\right) \cup\left(\bigcup_{a, b \in A} a \circ b\right) \cup\left(\bigcup_{a, b \in A} \bigcup_{a \in A_{1}} a \perp b\right. \\
& A_{2}=A_{1} \cup\left(\bigcup_{a, b \in A_{1}} a \circ b\right) \bigcup\left(\bigcup_{a, b \in A_{1}} a n d a \perp b\right. \\
& \ldots \\
& A_{n}=A_{n-1} \bigcup\left(\bigcup_{a \in A_{n-1}} a^{\prime}\right) \cup\left(\bigcup_{a, b \in A_{n-1}} a \circ b\right) \cup(\underbrace{\bigcup}_{a, b \in A_{n-1} \text { and } a \perp b} a \oplus b), \\
& \ldots \\
& \Gamma=\bigcup_{n=1}^{\infty} A_{n} . \\
& \text { Then } \bar{A}=\Gamma .
\end{aligned}
$$

Proof. First we prove that $\Gamma$ is a sub-sequential effect algebra of $(E, 0,1, \oplus, \circ)$.

If $a \in \Gamma$, then $a \in A_{n}$ for some $n$, so $a^{\prime} \in A_{n+1} \subseteq \Gamma$.

If $a, b \in \Gamma$, then $a, b \in A_{n}$ for some $n$, so $(a \circ b) \in A_{n+1} \subseteq \Gamma$.

If $a, b \in \Gamma$ and $a \perp b$, then $a, b \in A_{n}$ for some $n$, so $(a \oplus b) \in A_{n+1} \subseteq \Gamma$.

Thus, $\Gamma$ is closed under all the three operations $\oplus$, $\circ$ and '. So $\Gamma$ is a subsequential effect algebra of $(E, 0,1, \oplus, \circ)$.

Of course $A \subseteq \Gamma$. Since $\bar{A}$ is the smallest sub-sequential effect algebra of $(E, 0,1, \oplus, \circ)$ containing $A$, we have $\bar{A} \subseteq \Gamma$. On the other hand, by induction, it is easy to see that $A_{n} \subseteq \bar{A}$ for all $n$. Thus $\Gamma \subseteq \bar{A}$. So $\Gamma=\bar{A}$. 
Note that by using Theorem 2 we can also answer professor Gudder's problem by a constructive way, we omit the process.

\section{An addition property of sequential effect algebras}

Let $(E, 0,1, \oplus, \circ)$ be a sequential effect algebra, $a, b \in E$. If $\underbrace{a \oplus a \cdots \oplus a}_{\text {the number is } n}$ is defined, we denote it by na. Now, we are interested in the following uniqueness problem: If for some positive integer $n_{0} \geq 2, n_{0} a=n_{0} b$, then under what conditions $a=b$ hold? We have

Theorem 3. Let $(E, 0,1, \oplus, \circ)$ be a sequential effect algebra, $a, b \in E$ and for some positive integer $n_{0} \geq 2, n_{0} a=n_{0} b=c$. If $c \in E_{s}$ and $a \mid b$, then $a=b$.

Proof. Since $a \leq c$, by Lemma 1, $a=a \circ c$, similarly $b=b \circ c$.

By $(\mathrm{SEA} 1)$, we have $a \circ c=a \circ\left(n_{0} b\right)=n_{0}(a \circ b), b \circ c=b \circ\left(n_{0} a\right)=n_{0}(b \circ a)$.

Note that $a \mid b$, so $a \circ b=b \circ a$ and $a \circ c=b \circ c$. Thus $a=b$.

Now, we show that neither of the two conditions in Theorem 3 can be discarded.

Example 1. Let $I_{1}=[0,1], I_{2}=[0,1], E=H S\left(I_{1}, I_{2}\right)$ be the horizontal sum of $I_{1}, I_{2}$ (see [5, Section 8, the Example in $\left.P_{109}\right]$ ). For each $t \in[0,1]$, if it is in $I_{1}$, we denote it by $\hat{t}$; if it is in $I_{2}$, we denote it by $\check{t}$. Let $a=\frac{\hat{1}}{n_{0}}, b=\frac{\check{1}}{n_{0}}$. Then $n_{0} a=1=n_{0} b, 1 \in E_{s}, a \neq b, a \circ b \neq b \circ a$. So the condition $a \mid b$ in Theorem 3 can not be discarded.

Example 2. Let $\mathbf{N}$ be the nonnegative integer set, $n_{0}$ be a positive integer and $n_{0} \geq 2, E_{0}=\left\{0,1, a_{n, m}, b_{n, m} \mid n, m \in \mathbf{N}, n_{0}-1 \geq m, n^{2}+m^{2} \neq 0\right\}$.

First, we define a partial binary operation $\oplus$ on $E_{0}$ as follows (when we write $x \oplus y=z$, we always mean $x \oplus y=z=y \oplus x)$ :

For each $x \in E_{0}, 0 \oplus x=x$,

$$
a_{n, m} \oplus a_{r, s}= \begin{cases}a_{n+r, m+s}, & \text { if } m+s<n_{0} \\ a_{n+r+n_{0}, m+s-n_{0}}, & \text { if } m+s \geq n_{0}\end{cases}
$$




$$
a_{n, m} \oplus b_{r, s}= \begin{cases}b_{r-n, s-m}, & \text { if } n \leq r, m \leq s,(r-n)^{2}+(s-m)^{2} \neq 0 \\ 1, & \text { if } n=r, m=s \\ b_{r-n-n_{0}, s-m+n_{0}}, & \text { if } n+n_{0} \leq r, m>s .\end{cases}
$$

No other $\oplus$ operation is defined.

Next, we define a binary operation $\circ$ on $E_{0}$ as follows (when we write $x \circ y=z$, we always mean $x \circ y=z=y \circ x)$ :

For each $x \in E_{0}, 0 \circ x=0,1 \circ x=x$,

$$
a_{n, m} \circ a_{r, s}=0, a_{n, m} \circ b_{r, s}=a_{n, m},
$$

$$
b_{n, m} \circ b_{r, s}= \begin{cases}b_{n+r, m+s}, & \text { if } m+s<n_{0} ; \\ b_{n+r+n_{0}, m+s-n_{0}}, & \text { if } m+s \geq n_{0} .\end{cases}
$$

Now, we prove that $E_{0}$ is a sequential effect algebra.

In fact, (EA1) and (EA4) are trivial.

We verify (EA2), for simplicity, we omit the trivial cases about 0,1 :

$a_{k, j} \oplus\left(a_{n, m} \oplus a_{r, s}\right)=\left(a_{k, j} \oplus a_{n, m}\right) \oplus a_{r, s}$

$$
= \begin{cases}a_{k+r+n, s+j+m}, & \text { if } s+j+m<n_{0} ; \\ a_{k+r+n+n_{0}, s+j+m-n_{0}}, & \text { if } n_{0} \leq s+j+m<2 n_{0} \\ a_{k+r+n+2 n_{0}, s+j+m-2 n_{0}}, & \text { if } s+j+m \geq 2 n_{0} .\end{cases}
$$

Each $a_{k, j} \oplus\left(a_{n, m} \oplus b_{r, s}\right)$ or $\left(a_{k, j} \oplus a_{n, m}\right) \oplus b_{r, s}$ is defined if and only if one of the following four conditions is satisfied, at this case,

$$
a_{k, j} \oplus\left(a_{n, m} \oplus b_{r, s}\right)=\left(a_{k, j} \oplus a_{n, m}\right) \oplus b_{r, s}
$$

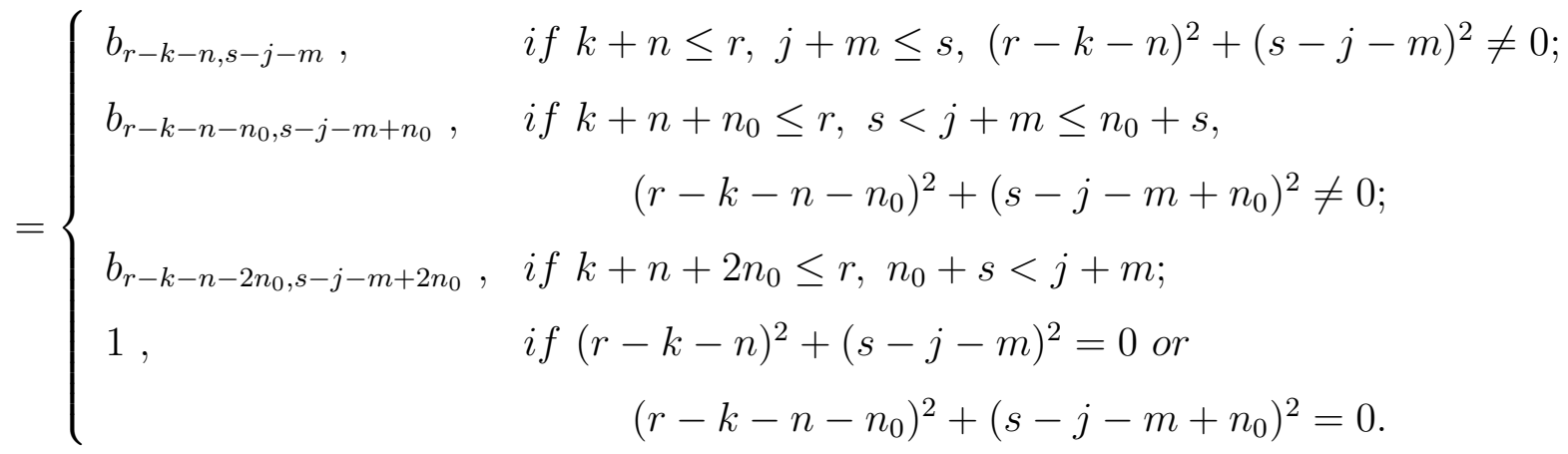


Thus, (EA2) is hold.

(EA3) is clear since $a_{n, m} \oplus b_{n, m}=1$. Thus, $\left(E_{0}, 0,1, \oplus\right)$ is an effect algebra.

Moreover, we verify that $\left(E_{0}, 0,1, \oplus, \circ\right)$ is a sequential effect algebra.

(SEA2) and (SEA3) and (SEA5) are trivial.

We verify (SEA1), for simplicity, we omit the trivial cases about 0,1 :

$a_{k, j} \circ\left(a_{n, m} \oplus a_{r, s}\right)=a_{k, j} \circ a_{n, m} \oplus a_{k, j} \circ a_{r, s}=0$.

$b_{k, j} \circ\left(a_{n, m} \oplus a_{r, s}\right)=b_{k, j} \circ a_{n, m} \oplus b_{k, j} \circ a_{r, s}= \begin{cases}a_{n+r, m+s}, & \text { if } m+s<n_{0} \\ a_{n+r+n_{0}, m+s-n_{0}}, & \text { if } m+s \geq n_{0} .\end{cases}$

When $a_{n, m} \oplus b_{r, s}$ is defined,

$a_{k, j} \circ\left(a_{n, m} \oplus b_{r, s}\right)=a_{k, j} \circ a_{n, m} \oplus a_{k, j} \circ b_{r, s}=a_{k, j}$,

$b_{k, j} \circ\left(a_{n, m} \oplus b_{r, s}\right)=b_{k, j} \circ a_{n, m} \oplus b_{k, j} \circ b_{r, s}$

$$
= \begin{cases}b_{r+k-n, s+j-m}, & \text { if } n \leq r, m \leq s, j+s<n_{0}+m ; \\ b_{r+k-n, s+j-m}, & \text { if } n+n_{0} \leq r, s<m \leq j+s ; \\ b_{r+k-n+n_{0}, s+j-m-n_{0}}, & \text { if } n \leq r, n_{0}+m \leq j+s ; \\ b_{r+k-n-n_{0}, s+j-m+n_{0}}, & \text { if } n+n_{0} \leq r, j+s<m .\end{cases}
$$

Thus, (SEA1) is true.

We verify (SEA4), for simplicity, we omit also the trivial cases about 0,1:

$$
\begin{aligned}
& a_{k, j} \circ\left(a_{n, m} \circ a_{r, s}\right)=\left(a_{k, j} \circ a_{n, m}\right) \circ a_{r, s}=0 . \\
& a_{k, j} \circ\left(a_{n, m} \circ b_{r, s}\right)=\left(a_{k, j} \circ a_{n, m}\right) \circ b_{r, s}=0 . \\
& a_{k, j} \circ\left(b_{n, m} \circ b_{r, s}\right)=\left(a_{k, j} \circ b_{n, m}\right) \circ b_{r, s}=a_{k, j} . \\
& b_{k, j} \circ\left(b_{n, m} \circ b_{r, s}\right)=\left(b_{k, j} \circ b_{n, m}\right) \circ b_{r, s} \\
& = \begin{cases}b_{k+r+n, s+j+m}, & \text { if } s+j+m<n_{0} ; \\
b_{k+r+n+n_{0}, s+j+m-n_{0}}, & \text { if } n_{0} \leq s+j+m<2 n_{0} ; \\
b_{k+r+n+2 n_{0}, s+j+m-2 n_{0}}, & \text { if } s+j+m \geq 2 n_{0} .\end{cases}
\end{aligned}
$$

Thus (SEA4) is hold and $\left(E_{0}, 0,1, \oplus, \circ\right)$ is a sequential effect algebra.

Finally, we show that the condition $c \in E_{s}$ in Theorem 3 can not be discarded. 
Indeed, since $a_{n, 0} \oplus a_{r, 0}=a_{n+r, 0}$, so $n_{0} a_{1,0}=a_{n_{0}, 0}$. Note that

$$
a_{0, m} \oplus a_{0, s}= \begin{cases}a_{0, m+s}, & \text { if } m+s<n_{0} ; \\ a_{n_{0}, m+s-n_{0}}, & \text { if } m+s \geq n_{0} .\end{cases}
$$

Thus, $\left(n_{0}-1\right) a_{0,1}=a_{0, n_{0}-1}, n_{0} a_{0,1}=\left(n_{0}-1\right) a_{0,1} \oplus a_{0,1}=a_{0, n_{0}-1} \oplus a_{0,1}=a_{n_{0}, 0}$, that is, $n_{0} a_{1,0}=a_{n_{0}, 0}=n_{0} a_{0,1}$. Note that $a_{n_{0}, 0} \circ a_{n_{0}, 0}=0$, so $a_{n_{0}, 0} \notin\left(E_{0}\right)_{s}$.

\section{References}

[1]. D. J. Foulis and M. K. Bennett: Effect algebras and unsharp quantum logics. Found. Phys. 24, 1331(1994).

[2]. S. Gudder: Sharply dominating effect algebras. Tatra Mt. Math. Publ. 15, 23(1998).

[3]. Z. Riecanova and J. D. Wu: States on sharply dominating effect algebras. Sci. in China A: Math. 51, 907(2008).

[4]. S. Gudder and G. Nagy: Sequential quantum measurements. J. Math. Phys. 42, 5212(2001).

[5]. S. Gudder and R. Greechie: Sequential products on effect algebras. Rep. Math. Phys. 49, 87(2002).

[6]. S. Gudder: Open problems for sequential effect algebras. Inter. J. Theory. Phys. 44, 2219(2005). 